\title{
A three-dimensional parabolic equation model of sound propagation using higher-order operator splitting and Padé approximants
}

\author{
Ying-Tsong Lin ${ }^{\text {a) }}$ \\ Applied Ocean Physics and Engineering Department, \\ Woods Hole Oceanographic Institution, Woods Hole, Massachusetts 02543 \\ ytlin@whoi.edu \\ Jon M. Collis \\ Department of Applied Mathematics and Statistics, Colorado School of Mines, \\ Golden, Colorado 80401 \\ jcollis@mines.edu \\ Timothy F. Duda \\ Applied Ocean Physics and Engineering Department, \\ Woods Hole Oceanographic Institution, Woods Hole, Massachusetts 02543 \\ tduda@whoi.edu
}

\begin{abstract}
An alternating direction implicit (ADI) three-dimensional fluid parabolic equation solution method with enhanced accuracy is presented. The method uses a square-root Helmholtz operator splitting algorithm that retains cross-multiplied operator terms that have been previously neglected. With these higher-order cross terms, the valid angular range of the parabolic equation solution is improved. The method is tested for accuracy against an image solution in an idealized wedge problem. Computational efficiency improvements resulting from the ADI discretization are also discussed.

(C) 2012 Acoustical Society of America

PACS numbers: 43.20.Bi, 43.30.Bp [WS]

Date Received: June 28, 2012 Date Accepted: August 29, 2012
\end{abstract}

\section{Introduction}

A method to employ the split-step Padé parabolic-equation (PE) method (Collins, 1993) to calculate the transmission loss (TL) of sound propagating in three-dimensional (3D) underwater environments is presented. In ocean acoustics, the split-step Padé method has been heavily used for two-dimensional (2D) vertical-plane calculations, where derivatives in the Helmholtz operator are evaluated in only one direction normal to the algorithm marching direction. In 3D applications, derivatives are evaluated in two normal directions, typically vertical and horizontal in ocean acoustic applications. The 3D Padé method can be applied directly by approximating the 2D derivatives (Collis, 2011). However, this would require great computational resources, even for a moderate model domain, because the approximations of $2 \mathrm{D}$ derivatives are not consistent with tridiagonal matrices that are easily inverted, stored, and updated.

As an alternative to the direct solution, the 2D derivative operator can be split into two one-dimensional (1D) operators for the vertical and horizontal derivatives, respectively. This operator splitting results in a tridiagonal formulation and allows a marching algorithm similar to the alternating direction implicit (ADI) method. This idea of operator splitting has been implemented for 3D PE methods, for example, by Lee and Schultz (1995) and Sturm (2005), but the previous work neglected cross-

\footnotetext{
${ }^{\text {a) }}$ Author to whom correspondence should be addressed.
} 
multiplied terms of the two $1 \mathrm{D}$ operators, with a consequence of reduced wide-angle $\mathrm{PE}$ accuracy, as noted by Sturm (2005). To improve accuracy, a multi-directional scheme (Collino and Joly, 1995) to split the transverse 2D operator into four 1D operators along the vertical, horizontal, and two diagonal directions could be utilized, but this will require square cell geometry in the model, which is undesirable. The alternative presented here is an iterative scheme to directly incorporate the required (higher order) cross terms into the PE solution that does not constrain the grid geometry and uses only the vertical and horizontal derivative operators, and is thus consistent with tridiagonal matrices. The accuracy of the method is demonstrated with a $3 \mathrm{D}$ wedge problem.

\section{Higher-order operator splitting}

Consider the following 3D Helmholtz wave equation in Cartesian coordinates $(x, y, z)$

$$
\rho \frac{\partial}{\partial x}\left(\frac{1}{\rho} \frac{\partial}{\partial x}\right) p+\rho \frac{\partial}{\partial y}\left(\frac{1}{\rho} \frac{\partial}{\partial y}\right) p+\rho \frac{\partial}{\partial z}\left(\frac{1}{\rho} \frac{\partial}{\partial z}\right) p+k^{2} p=0
$$

where $p$ is the sound pressure, $\rho$ is the medium density, and $k$ is the medium wavenumber. The advantage of using Cartesian coordinates is on achieving uniform model resolution with a fixed discretization mesh. The sound pressure variable can be further reduced to $u=p \exp \left(-i k_{0} x\right) / \alpha$, where the baseline phase of $p$ is removed according to a reference wavenumber $k_{0}$, and $\alpha$ can be any function related to medium properties. The reference wavenumber $k_{0}$ is defined to be $\omega / c_{0}$, where $\omega$ is the angular frequency of sound and $c_{0}$ is a given reference sound speed. To derive a one-way parabolic wave equation along the $x$-axis, we first approximate the $x$-dependent environment by a series of $x$-independent regions. In each of these regions, the one-way parabolic wave equation of $u$ is

$$
\frac{\partial u}{\partial x}=i k_{0}\left\{-1+\sqrt{1+\left(n^{2}-1\right)+k_{0}^{-2} \frac{\rho}{\alpha} \frac{\partial}{\partial y}\left(\frac{1}{\rho} \frac{\partial}{\partial y} \alpha\right)+k_{0}^{-2} \frac{\rho}{\alpha} \frac{\partial}{\partial z}\left(\frac{1}{\rho} \frac{\partial}{\partial z} \alpha\right)}\right\} u,
$$

where $n=k / k_{0}$ is the refractive index with respect to $k_{0}$. Because Eq. (2) is an evolution equation with respect to $x$, it can be solved using a range marching algorithm with a continuity condition enforced at the vertical interface connecting the solution at the next step. To conserve energy at each marching step, we let $\alpha$ be $\sqrt{\rho c}$ (Collins and Westwood, 1991), where $c$ is the medium sound speed. To simplify notation, the square-root Helmholtz operator in Eq. (2) is denoted by $\sqrt{1+\mathcal{Y}+\mathcal{Z}}$ with $\mathcal{Y}$ and $\mathcal{Z}$ defined as

$$
\begin{gathered}
\mathcal{Y}=w\left(n^{2}-1\right)+k_{0}^{-2} \frac{\rho}{\alpha} \frac{\partial}{\partial y}\left(\frac{1}{\rho} \frac{\partial}{\partial y} \alpha\right), \\
\mathcal{Z}=(1-w)\left(n^{2}-1\right)+k_{0}^{-2} \frac{\rho}{\alpha} \frac{\partial}{\partial z}\left(\frac{1}{\rho} \frac{\partial}{\partial z} \alpha\right),
\end{gathered}
$$

where $w$ is a weighting factor between 0 and 1 to control the splitting of sound speed anomalies into $\mathcal{Y}$ and $\mathcal{Z}$. The weighting factor used here, of our own design, is related to the ratio of the grid increments in $y$ and $z$ via $w=\Delta z /(\Delta y+\Delta z)$.

The solution of Eq. (2) can be written formally as

$$
u(x+\Delta x)=e^{\delta[-1+\sqrt{1+\mathcal{Y}+\mathcal{Z}}]} u(x)+E,
$$

where $\delta=i k_{0} \Delta x$. The error $E$ is of order $(\Delta x)^{2}$, and it is due to the $x$-independent approximation applied to the environmental model within each solution marching step from $x$ to $x+\Delta x$ (Jensen et al., 1994). Equation (4) can be solved directly by approximating the exponential operator with a 2D Padé expansion (Collis, 2011), but it may 
require large computation resources, as will be demonstrated in a numerical example. In order to allow more efficient numerical schemes, the square-root operator can be split into two 1D operators as

$$
\sqrt{1+\mathcal{Y}+\mathcal{Z}} \cong-1+\sqrt{1+\mathcal{Y}}+\sqrt{1+\mathcal{Z}}=\mathcal{Q}_{2},
$$

which is denoted by $\mathcal{Q}_{2}$ since the standard PE approximation is $\mathcal{Q}_{1}=1+\mathcal{Y} / 2+\mathcal{Z} / 2$. The split operator $\mathcal{Q}_{2}$ has been used in previous 3D ADI PE applications, e.g., Lee and Schultz (1995) and Sturm (2005), where cylindrical coordinates were utilized with the horizontal operator $\mathcal{Y}$ acting in the azimuth. Later in this section, it will be shown that $\mathcal{Q}_{2}$ is only valid for small propagation angles. To achieve a wide-angle capability, we introduce two cross terms with $\mathcal{Y}$ and $\mathcal{Z}$ in the splitting as shown below.

$$
\begin{aligned}
\sqrt{1+\mathcal{Y}+\mathcal{Z}} \cong & -1+\sqrt{1+\mathcal{Y}}+\sqrt{1+\mathcal{Z}}-(-1+\sqrt{1+\mathcal{Y}})(-1+\sqrt{1+\mathcal{Z}}) / 2 \\
& -(-1+\sqrt{1+\mathcal{Z}})(-1+\sqrt{1+\mathcal{Y}}) / 2=\mathcal{Q}_{3},
\end{aligned}
$$

which is obtained from a second-order Taylor expansion around $\sqrt{1+\mathcal{Y}}=1$ and $\sqrt{1+\mathcal{Z}}=1$, and the non-commutativity of $\mathcal{Y}$ and $\mathcal{Z}$ is kept in the formulation. This operator splitting scheme, denoted by $\mathcal{Q}_{3}$, is used by Lin and Duda (2012) for a higher order split-step Fourier PE method, where, unlike the current application, the squareroot Helmholtz operator is split into two operators to take care of free propagation and refractive index anomalies. One way to handle the bottom interface condition in the split-step Fourier method is to apply interface smoothing, and with the previous operator splitting schemes $\left(\mathcal{Q}_{1}\right.$ and $\left.\mathcal{Q}_{2}\right)$ the smoothing width for the density profile needs to be in an order of $1 / 5$ th of an acoustic wavelength, as suggested in Jensen et al. (1994). Now, with the new operator splitting $\mathcal{Q}_{3}$, the smoothing width can be as small as 1/15th of a wavelength (Lin and Duda, 2012), which is $1.25 \mathrm{~m}$ at $75 \mathrm{~Hz}$, and it indeed reduces the model errors caused by the smoothing procedure. However, for a very low frequency, the smoothing width is still too large, e.g., $4 \mathrm{~m}$ at $25 \mathrm{~Hz}$, to keep the model errors down, and it limits the low-frequency applications of the Fourier method. In this paper, we will demonstrate that the split-step Padé method can well handle the bottom interface without smoothing it.

We shall now examine the phase errors that may be produced by the higher-order split operator $Q_{3}$. Consider a wavenumber vector $\vec{k}=k_{x} \hat{e}_{x}+k_{y} \hat{e}_{y}+k_{z} \hat{e}_{z}=k_{0}\left(\cos \theta \hat{e}_{x}\right.$ $+\sin \theta \cos \phi \hat{e}_{y}+\sin \theta \sin \phi \hat{e}_{z}$ ), where $\theta$ is the inclination angle from the $x$ axis, and $\phi$ is the orientation angle from the $y$ axis on the vertical $y-z$ plane. Following a standard error analysis for PE methods (Jensen et al., 1994), the relative phase errors of $\mathcal{Q}_{2}$ and $\mathcal{Q}_{3}$ are

$$
\varepsilon_{2}=\left|-1+\cos \theta-\varepsilon_{y}-\varepsilon_{z}\right|,
$$

and

$$
\varepsilon_{3}=\left|-1+\cos \theta-\varepsilon_{y}-\varepsilon_{z}+\varepsilon_{y} \varepsilon_{z}\right|,
$$

where $\varepsilon_{y}=-1+\sqrt{1-\sin ^{2} \theta \cos ^{2} \phi}$ and $\varepsilon_{z}=-1+\sqrt{1-\sin ^{2} \theta \sin ^{2} \phi}$. The relative phase errors in Eq. (7) are defined by $|\Delta \tau / \tau|$, where $\tau$ is the correct phase and $\Delta \tau$ indicates the phase error. When the wavenumber vector $\vec{k}$ is orientated to line up with either the $y$ or $z$ axis, i.e., $\phi=0, \pm \pi / 2$ or $\pi$, both $\varepsilon_{2}$ and $\varepsilon_{3}$ will vanish. This indicates that a perfect operator splitting only occurs when there is no cross component of $k_{y}$ and $k_{z}$ in a wave vector. In addition, it can be shown that for a given inclination angle $\theta$, both errors have their maxima when the wavenumber vector is orientated to the diagonals of the $y-z$ plane. So, if we consider an error tolerance of $1 \times 10^{-4}$ and take $\phi$ equal to $\pi / 4$, the valid angle using $\mathcal{Q}_{2}$ is bounded by $11.5^{\circ}$ measured from the $x$ axis; the corresponding angles using $\mathcal{Q}_{3}$ can extend to $22^{\circ}$, which is an improvement of nearly double. If we allow a greater error tolerance, say $1 \times 10^{-3}$, the 
valid angle can be even as large as $32.5^{\circ}$. This error analysis shows the wide-angle capability of this higher order splitting method, and an iteration scheme is proposed next to calculate the corresponding PE solution.

\section{Split-step Padé solutions}

The PE solution with the higher order operator splitting $Q_{3}$ can be determined from

$$
\begin{aligned}
& u(x+\Delta x) \cong e^{\delta(-1+\sqrt{1+\mathcal{Y}})} e^{\delta(-1+\sqrt{1+\mathcal{Z}})} \\
& \times e^{-\delta / 2[(-1+\sqrt{1+\mathcal{Y}})(-1+\sqrt{1+\mathcal{Z}})+(-1+\sqrt{1+\mathcal{Z}})(-1+\sqrt{1+\mathcal{Y}})]} \mathcal{u}(x),
\end{aligned}
$$

and the exponentiated cross operator can be implemented with a Taylor expansion

$$
\begin{aligned}
& \exp \left\{-\frac{\delta}{2}[(-1+\sqrt{1+\mathcal{Y}})(-1+\sqrt{1+\mathcal{Z}})+(-1+\sqrt{1+\mathcal{Z}})(-1+\sqrt{1+\mathcal{Y}})]\right\} \\
& =1+\sum_{m=1}^{\infty} \frac{1}{m !}\left\{-\frac{\delta}{2}[(-1+\sqrt{1+\mathcal{Y}})(-1+\sqrt{1+\mathcal{Z}})+(-1+\sqrt{1+\mathcal{Z}})(-1+\sqrt{1+\mathcal{Y}})]\right\}^{m}
\end{aligned}
$$

which will be truncated to a certain order $M$ depending on the required level of precision. The detailed steps to calculate the solution are outlined in the following:

$$
\begin{gathered}
\Delta u_{1}=-\frac{\delta}{2}[(-1+\sqrt{1+\mathcal{Y}})(-1+\sqrt{1+\mathcal{Z}})+(-1+\sqrt{1+\mathcal{Z}})(-1+\sqrt{1+\mathcal{Y}})] u(x), \\
\Delta u_{m}=-\frac{\delta}{2 m}[(-1+\sqrt{1+\mathcal{Y}})(-1+\sqrt{1+\mathcal{Z}})+(-1+\sqrt{1+\mathcal{Z}})(-1+\sqrt{1+\mathcal{Y}})] \Delta u_{m-1} \text { for } m>1 \\
u(x+\Delta x)=e^{\delta(-1+\sqrt{1+\mathcal{Y}})} e^{\delta(-1+\sqrt{1+\mathcal{Z}})}\left[\sum_{m=1}^{M} \Delta u_{m}+u(x)\right]
\end{gathered}
$$

where Eqs. (10a) and (10b) are used to calculate higher-order corrections from the cross-term operators, and the corrections are added to the solution at $x$ before marching to $x+\Delta x$ as shown in Eq. (10c). One important feature of this iteration scheme is that the partial derivatives with respect to $y$ and $z$ are implemented sequentially in pairs. Thus, for each $\partial y$ or $\partial z$ operator the system of finite difference equations will be tridiagonal and easily solved.

The square-root operators in the PE solution can be manipulated through the Padé approximations. First, we follow Collins (1993) to expand the exponentiated square-root operators in Eq. (10c) into a sequential product of rational operators

$$
e^{\delta(-1+\sqrt{1+\mathcal{G}})}=\prod_{l=1}^{L} \frac{1+\alpha_{l, L} \mathcal{G}}{1+\beta_{l, L} \mathcal{G}},
$$

which is a Padé approximant of order $L$, and $\alpha_{l, L}$ and $\beta_{l, L}$ are the coefficients. In the equation, the operator $\mathcal{G}$ can be $\mathcal{Y}$ or $\mathcal{Z}$ depending on which derivatives are being used. As for the square-root operators in Eqs. (10a) and (10b), we use the rotated complex Padé approximant derived by Milinazzo et al. (1997), which can be written into a series of rational operators 


$$
-1+\sqrt{1+\mathcal{G}}=\sum_{l=1}^{L} \frac{a_{l, L} \mathcal{G}}{1+b_{l, L} \mathcal{G}}
$$

Finally, when the evanescent sound pressure field is considered in the Taylor expansion of the exponentiated cross operator, Eq. (9), it will require a small marching step for the expansion to converge. To prevent the PE solution from diverging with a large marching step, a low pass filter can be applied to the solution to remove its high wavenumber evanescent component after each square-root operator is implemented. The low pass filter is like a stability constraint, and some portion of the evanescent spectrum can be passed to maintain the accuracy of the solution.

\section{Numerical examples}

An idealized wedge problem shown in Fig. 1(a) is considered here. The slope angle is $\pi / 63 \mathrm{rad}\left(\sim 2.86^{\circ}\right)$, and a $25-\mathrm{Hz}$ point source is located $4 \mathrm{~km}$ away from the wedge apex at $100 \mathrm{~m}$ depth. The water column is homogeneous with sound speed $1500 \mathrm{~m} / \mathrm{s}$, density $1 \mathrm{~g} / \mathrm{cm}^{3}$, and no medium loss. The bottom is also homogeneous with sound speed $1700 \mathrm{~m} / \mathrm{s}$ and medium attenuation $0.5 \mathrm{~dB}$ per wavelength. Two examples with bottom density equal to 1 or $1.5 \mathrm{~g} / \mathrm{cm}^{3}$ will be presented.

In the first example, we consider bottom density to be $1 \mathrm{~g} / \mathrm{cm}^{3}$. Because there is no density contrast and the environment does not vary along the PE marching direction ( $x$ axis), we do not need to use the $\alpha$-reduced pressure variable shown in Eq. (2). A standard second-order central finite difference scheme is adequate to provide a stable model discretization with a horizontal grid size $\Delta y=2 \mathrm{~m}$ and a depth grid size $\Delta z=1 \mathrm{~m}$. The PE marching step $\Delta x$ is $10 \mathrm{~m}$ with a reference sound speed $c_{0}=1500 \mathrm{~m} / \mathrm{s}$. Artificial absorption
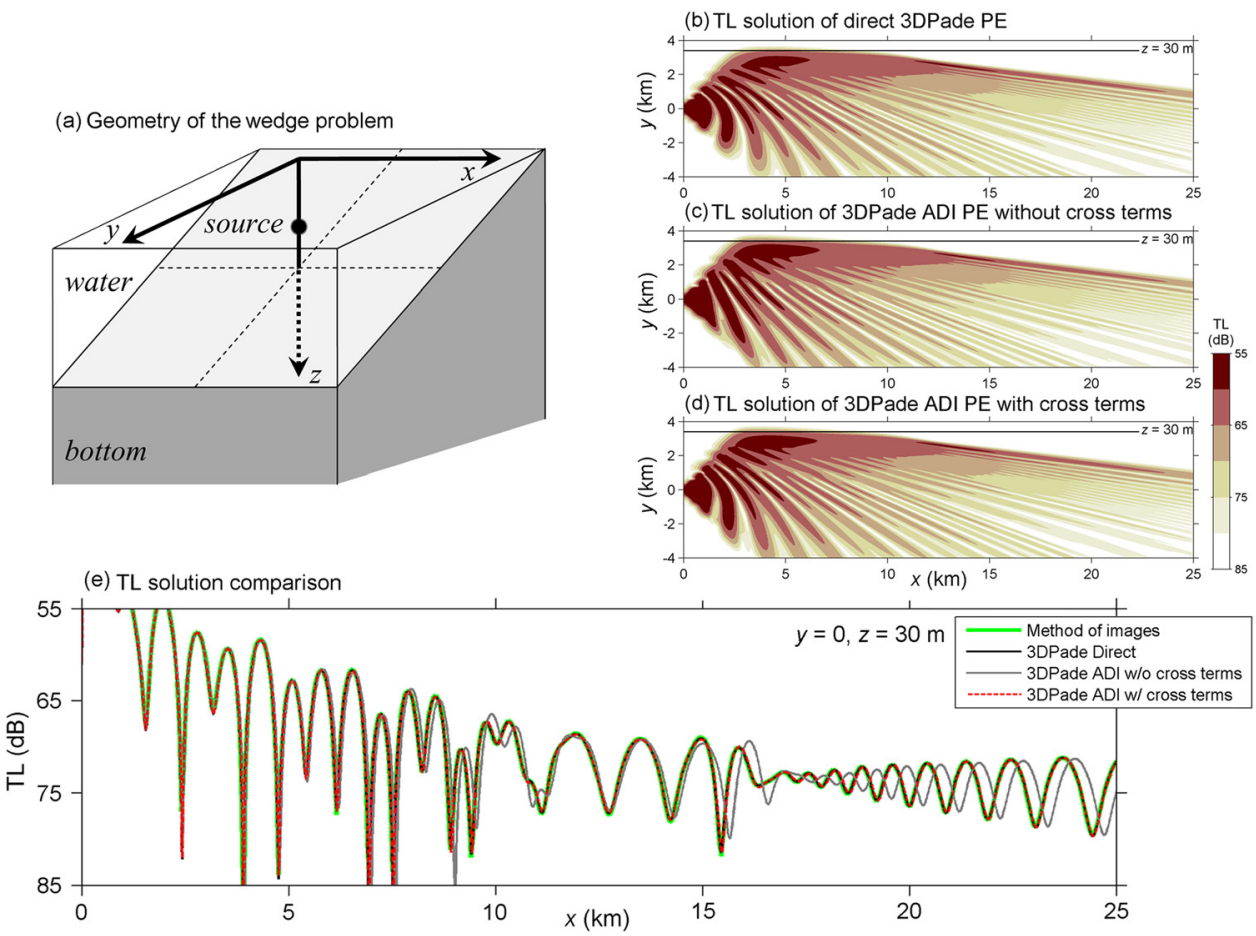

Fig. 1. (Color online) Modeling of 3D sound propagation in an idealized wedge with bottom density equal to $1 \mathrm{~g} / \mathrm{cm}^{3}$. (a) Model geometry. (b)-(d) TL solutions in the $x-y$ plane at $z=30 \mathrm{~m}$ obtained from three different Padé methods. (e) Comparison of the three Padé solutions with the image solution along the line at $y=0$ and $z=30 \mathrm{~m}$. 
layers are implanted on the horizontal and bottom boundaries of the computational domain at $y= \pm 4125 \mathrm{~m}$ and $z=562 \mathrm{~m}$ to imitate radiation conditions. The thickness of the artificial absorption layers are 1375 and $188 \mathrm{~m}$ on the horizontal and vertical boundaries, respectively. Three different Padé PE solutions are calculated and compared to an image solution derived by Deane and Buckingham (1993). Since the slope angle is an integer submultiple of $\pi$, there is no imaginary source image. The three Padé PE solutions are shown in Figs. 1(b)-1(d), including: (1) The direct solution obtained by solving the full exponentiated square-root Helmholtz operator in Eq. (4), (2) the ADI solution without cross terms, and (3) with cross terms. The exponentiated square-root Helmholtz operators, either the full one for the direct solution or the split ones for the ADI solution, are expanded by twoterm Padé approximants, see Eq. (11). The cross-term corrections for the ADI solution are calculated using three-term rotated Padé approximants with a low pass filter cut at $2.5 k_{0}$, see Eq. (12), and the corrections take no more than four iterations to reach the required precision, $\left|\Delta u_{m}\right| /|u| \leq 1 \times 10^{-4}$. The direct solution shown in Fig. 1(b) is most accurate because no operator splitting is taken place. This can be confirmed from the agreement to the reference solution shown in Fig. 1(e). The 3D Padé ADI solution without the cross-term corrections in Fig. 1(c) suffers from severe phase errors, and, as a result, no curvature is observed in the horizontal interference pattern of the solution. The comparison to the reference solution in Fig. 1(e) is indeed poor, especially for $x \geq 15 \mathrm{~km}$. To reduce the phase errors in the ADI solution, the cross-term corrections have to be made and result in a curved interference pattern as seen in Fig. 1(d). The agreement to the reference solution is indeed improved significantly in Fig. 1(e). Note that when the alternatingdirection method is implemented in a cylindrical coordinate system, the solution without cross-term corrections will have curvature due to the nature of the coordinate system, but the phase errors will still be significant, as seen in Fig. 7 of Sturm (2005).

It is worth noting the computational resources that the two different Padé methods required in the first example. With a second-order central difference scheme, the 2D second derivatives will result in banded matrices with five diagonals. In the current case with a model domain of 5500 points $\times 750$ points and utilizing two-term Padé approximants, nearly 30 gigabytes of computer memory are required to implement matrix inversions in the direct 3D Padé method. On the other hand, we only need about 3 gigabytes in the ADI method. A factor of ten reduction in computer memory requirement is achieved. An analysis of the relative computational speeds has also been performed using the model domain given above. In а МАTLAB R2011a double precision programming environment, using one central-processing-unit core on an Intel ${ }^{\circledR}$ Xeon $^{\circledR}$ X5492 Quad-core Processor, the Padé ADI method can complete one marching step in $54 \mathrm{~s}$. The direct Padé method needs $441 \mathrm{~s}$ to calculate the inverse of the banded matrices for the PE marching algorithm (a factor of eight difference). In addition, the higher-order split-step Fourier method (Lin and Duda, 2012) takes $24 \mathrm{~s}$ for this step. So the three relative speeds are 1,8, and 16. Note that all of these methods can benefit from parallel computing. This has been implemented in the Fourier method and will be done for the ADI method in the future.

In the second example, we consider a bottom density of $1.5 \mathrm{~g} / \mathrm{cm}^{3}$ and keep the other model settings the same as in the first example. A Galerkin method introduced by Collins and Westwood (1991) is used to discretize the 1D derivative operators in the ADI method. We also employ an impedance-reduced pressure variable, i.e., $\alpha=\sqrt{\rho c}$ in Eq. (2), to conserve energy at each marching step. Since the Galerkin discretization of 2D second derivatives results in banded matrices with nine diagonals (Collis, 2011), which overloads the computational resources for the current study, we only implement the ADI method and examine solutions obtained with and without cross-term corrections. The method of images by Deane and Buckingham (1993) is again used to compute a reference solution, and Fig. 2 shows that the ADI solution without cross-term corrections has significant phase errors as discussed in the previous example. We use three-term rotated Padé approximants with a low pass filter cut at $2.5 k_{0}$ to calculate the cross-term corrections for the ADI solution, and the improvement is noticeable, see Fig. 2. The phase errors are corrected, and only small disagreements in the magnitude are observed, which are due to the low pass filter applied in 


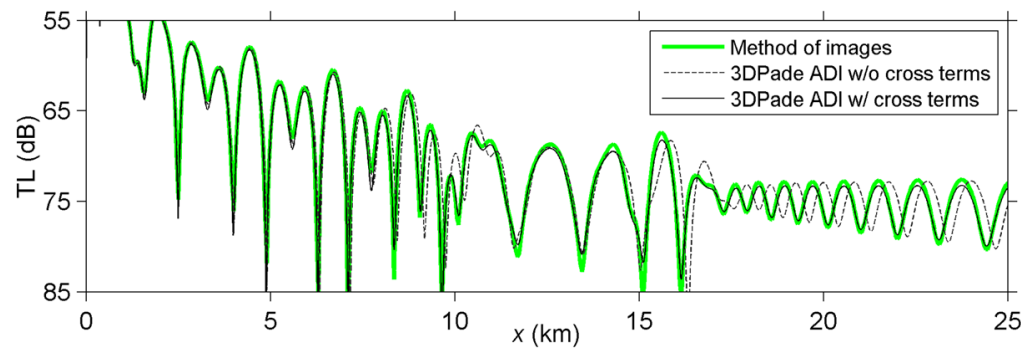

Fig. 2. (Color online) Comparison of three different TL solutions in an idealized wedge. The model geometry is as shown in Fig. 1(a), but the bottom density is set to $1.5 \mathrm{~g} / \mathrm{cm}^{3}$.

calculating the cross-term corrections. Although the low pass filter helps convergence of the final solution, it may still sacrifice some accuracy depending on the impedance of the bottom. Recall that in the previous example where there is no density contrast in the medium, the low pass filter did not cause a significant loss of solution accuracy.

\section{Summary}

An improved ADI PE method for modeling 3D underwater sound propagation has been presented and benchmarked. This method employs a higher-order scheme to split the square-root Helmholtz operator and results in a new PE factorization that handles greater propagation angles. The new solution has been benchmarked against an image solution for a 3D wedge problem. This benchmark also demonstrates the necessity of including the operator cross terms in ADI types of 3D PE approaches. Furthermore, the new solution lends itself to a model discretization that enables efficient tridiagonalmatrix inversion, and can be computed using existing desktop computer resources.

\section{Acknowledgments}

This work was sponsored by the Office of Naval Research under Grant Nos. N00014-101-0040 and N00014-11-1-0701. The authors also thank Arthur Newhall of the Woods Hole Oceanographic Institution for his valuable comments on the manuscript.

\section{References and links}

Collino, F., and Joly, P. (1995). "Splitting of operators, alternate directions, and paraxial approximations for the three-dimensional wave equation," SIAM J. Sci. Comput. (USA) 16, 1019-1048.

Collins, M. D. (1993). “A split-step Padé solution for parabolic equation method,” J. Acoust. Soc. Am. 93, 1736-1742.

Collins, M. D., and Westwood, E. K. (1991). "A higher-order energy-conserving parabolic equation for range-dependent ocean depth, sound speed, and density," J. Acoust. Soc. Am. 89, 1068-1075.

Collis, J. M. (2011). "Three-dimensional underwater sound propagation using a split-step Padé parabolic equation solution (A),” J. Acoust. Soc. Am. 130, 2528.

Deane, G. B., and Buckingham, M. J. (1993). "An analysis of the three-dimensional sound field in a penetrable wedge with a stratified fluid or elastic basement," J. Acoust. Soc. Am. 93, 1319-1328.

Jensen, F. B., Kuperman, W. A., Porter, M. B., and Schmidt, H. (1994). Computational Ocean Acoustics (AIP, New York), Chap. 6.

Lee, D., and Schultz, M. H. (1995). Numerical Ocean Acoustic Propagation in Three Dimensions (World Scientific, Singapore), Chap. 4.

Lin, Y.-T., and Duda, T. F. (2012). "A higher-order split-step Fourier parabolic-equation sound propagation solution scheme,” J. Acoust. Soc. Am. 132, EL61-EL67.

Milinazzo, F. A., Zala, C. A., and Brook, G. H. (1997). "Rational square-root approximation for parabolic equation algorithms," J. Acoust. Soc. Am. 101, 760-766.

Sturm, F. (2005). "Numerical study of broadband sound pulse propagation in three-dimensional oceanic waveguides," J. Acoust. Soc. Am. 117, 1058-1079. 